\title{
Association of the human papillomavirus infection with the recurrence of sinonasal inverted papilloma: a systematic review and meta-analysis*
}

\author{
Min-Seok Rha', Chang-Hoon Kim 1,2,3,4, Joo-Heon Yoon ${ }^{1,2,3,5}$, Hyung-Ju Cho 1,2,3 Rhinology 60: 1, 2- 10, 2022 \\ https://doi.org/10.4193/Rhin21.255 \\ ' Department of Otorhinolaryngology, Yonsei University College of Medicine, Seoul, Republic of Korea \\ ${ }^{2}$ The Airway Mucus Institute, Yonsei University College of Medicine, Severance Hospital, Seoul, Republic of Korea \\ *Received for publication: \\ ${ }^{3}$ The Korea Mouse Phenotyping Center, Yonsei University College of Medicine, Seoul, Republic of Korea \\ July 14, 2021 \\ ${ }^{4}$ Taste Research Center, Yonsei University College of Medicine, Seoul, Republic of Korea \\ Accepted: October 19, 2021 \\ ${ }^{5}$ Global Research Laboratory for Allergic Airway Diseases, Seoul, Republic of Korea
}

\begin{abstract}
Background: Although the role of human papillomavirus (HPV) in sinonasal inverted papilloma (SNIP) has been investigated, the link between HPV infection and SNIP recurrence remains controversial. This meta-analysis aimed to investigate the association between HPV infection and recurrence of SNIP.
\end{abstract}

Methods: The PubMed, Web of Science, Google Scholar, and Cochrane Library databases were searched (until 16 June 2021) to collect all relevant articles. The pooled odds ratio (OR) with 95\% confidence interval (Cl) was calculated using the fixed effects model. In addition, subgroup analysis, assessment of publication bias, and sensitivity analyses were performed.

Results: Fourteen eligible articles, including 592 patients with SNIP, were included in this study. Pooled analysis revealed that HPVpositive cases exhibited a significantly higher OR of tumour recurrence than HPV-negative counterparts. A significant association between HPV infection and tumour recurrence remained stable in subgroup analyses according to publication year of studies.

Conclusions: Our meta-analysis demonstrates that HPV infection is significantly associated with the recurrence of SNIP, suggesting the pathological role of HPV in SNIP. These results suggest that HPV infection should be considered in the management of SNIP.

Key words: human papillomavirus, HPV, inverted papilloma, sinonasal inverted papilloma, recurrence

\section{Introduction}

Sinonasal inverted papilloma (SNIP) is one of the most common benign tumours of the nose and paranasal sinuses, representing up to $4 \%$ of primary sinonasal tumours ${ }^{(1)}$. SNIP preferentially affects men, and its typical clinical symptom is unilateral nasal obstruction (1). The treatment of SNIP is challenging due to its local invasiveness, high recurrence rate, and substantial potential for malignant transformation. Squamous cell carcinoma (SCCa) may occur synchronously or metachronously with SNIP (2). Several factors, including human papillomavirus (HPV) infection, chronic inflammation, smoking, and occupational irritants, have been proposed to be associated with the development of SNIP
(3). However, the aetiology and precise mechanisms underlying the development of SNIP remain unclear.

$\mathrm{HPV}$ is a double-stranded DNA virus infecting epithelial cells of skin and mucosa ${ }^{(4)}$. In certain circumstances, persistent HPV infection induces uncontrolled growth of host cells, thereby leading to various diseases, from skin warts to cancer of diverse tissues ${ }^{(5)}$. In particular, the infection with high-risk HPV strains, such as HPV 16 and HPV 18, results in malignant transformation by the interaction between viral proteins and host cellular proteins ${ }^{(5,6)}$. In fact, HPV infection is a primary risk factor for the development of cervical cancer ${ }^{(5,7)}$. In the upper aerodigestive tract, HPV is known to cause oropharyngeal SCCa ${ }^{(8)}$. Over the 
past several decades, the incidence of HPV-related oropharyngeal SCCa has increased gradually ${ }^{(9)}$.

Although many studies have identified HPV infection in SNIP, the causative role of HPV infection in the development of SNIP is under debate. In particular, previous studies have reported controversial results regarding the relationship between HPV infection and recurrence of SNIPs ${ }^{(10-23)}$. Some researchers have reported that HPV infection is significantly associated with recurrence of SNIP (10-14,19,23), whereas others did not ${ }^{(15-18,20-22)}$. Contradictory results may be due to multiple factors, including the study design, HPV detection method, statistical power, genetic heterogeneity of the study population, and geographic factors. Therefore, we performed a systematic review and meta-analysis of relevant studies to determine whether HPV infection is associated with the recurrence of SNIP.

\section{Methods}

\section{Search strategy}

We conducted this systematic review in adherence to the guidelines of the Preferred Reporting Items for Systematic Reviews and Meta-Analyses ${ }^{(24)}$. A systematic search of publications in the PubMed, Web of Science, Google Scholar, and Cochrane Library electronic databases (until 16 June 2021) was conducted using the following MeSH terms and keywords: "Papilloma, Inverted" [MeSH], "inverted papilloma", "sinonasal", "Papillomaviridae"[MeSH], "human papillomavirus", and "HPV". The full search strategy is presented in Sup-Table 1.

Eligibility criteria, study selection, and quality assessment The population, intervention, comparator, outcome, and study design (PICOS) approaches were utilised to define study eligibility. 1) P: patients with pathologically confirmed SNIP; 2) I: detection of HPV DNA; 3) C: SNIP patients with or without HPV infection; 4) O: the odds ratio (OR) of tumour recurrence; 5) S: randomised controlled trials or observational studies including cross-sectional studies, cohort studies, case-control studies, or case series. In addition, studies were eligible if they were fulltext publications written in English. The abstracts were independently screened for relevance by two investigators. The full texts of the screened articles were reviewed independently by two authors. If studies did not provide information on HPV positivity and the presence of recurrence, they were excluded. The quality of each study was assessed using the modified Newcastle-Ottawa scale (NOS) ${ }^{(25)}$, with a maximum of seven points for observational studies based on the following components: patient selection, comparability, and outcome (Sup-Table 2). Two researchers independently evaluated the quality of each study. Disagreements between researchers were resolved through consensus. Studies with five or more stars were considered high-quality studies. Quality assessment of the included studies is presented in Sup-Table 2.

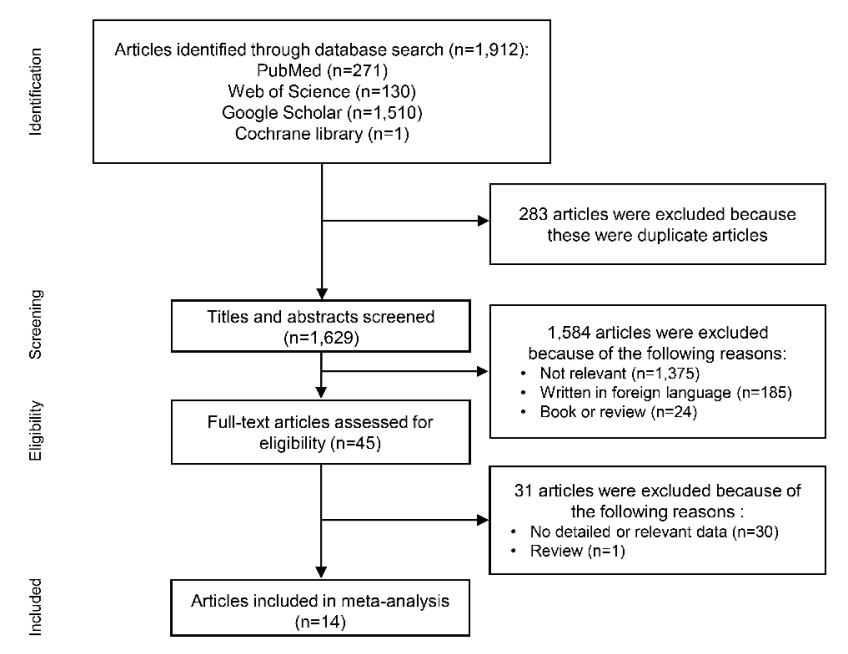

Figure 1. Flow diagram of the study selection.

\section{Data extraction}

The extracted information was as follows: author information, publication year, geographic location of studies, study design, sample number, method of HPV detection, HPV detection rate, mean follow-up period, and presence of tumour recurrence. Data regarding sample number, $\mathrm{HPV}$ detection rate, and tumour recurrence were extracted from cases of SNIP without carcinoma; however, not from those of SNIP with carcinoma. Tumour recurrence was defined as either "tumour recurrence during the follow-up period after the HPV test" or "a recurrent tumour at the time of HPV test". When the studies provided data regarding the follow-up period as a median and range (or interquartile range), the mean value was estimated using the following equation (26):

Mean $=\frac{(\text { min. }+ \text { max. }+2 \times \text { median })}{4}$ or $\frac{(\mathrm{q} 1+\mathrm{q} 3+\text { median })}{4}$

\section{Statistical analysis}

The meta-analysis was performed using $R$ 4.1.0 version statistical software (R Foundation for Statistical Computing, Vienna, Austria. URL https://www.R-project.org/.). The pooled ORs of SNIP recurrence and $95 \%$ confidence interval $(\mathrm{Cl})$ were calculated. Heterogeneity across the enrolled studies was calculated using the $\mathrm{I}^{2}$ test. Outcomes without a significant level of heterogeneity $\left(I^{2}<50\right)$ were analysed using the fixed effects model. In cases with $\mathrm{I}^{2}>50 \%$, which indicates significant heterogeneity between studies, the random effects model was used to generate pooled outcomes. Subgroup analysis was performed to evaluate the effects of the HPV detection method, geographic region of studies, and publication year of the studies. A funnel plot and Egger's test were used to detect publication bias ${ }^{(27)}$. Sensitivity analyses were performed to estimate the influence of each study on the overall meta-analysis results ${ }^{(28)}$. 
Table 1. Characteristics of the included studies.

\begin{tabular}{|c|c|c|c|c|c|c|c|c|c|c|}
\hline \multirow[t]{2}{*}{ Study } & \multirow[t]{2}{*}{ Year } & \multirow[t]{2}{*}{ Country/region } & \multirow[t]{2}{*}{ NOS } & \multirow{2}{*}{$\begin{array}{l}\text { HPV detection } \\
\text { method }\end{array}$} & \multirow{2}{*}{$\begin{array}{c}\text { Mean F/U } \\
\text { period } \\
\text { (months) }\end{array}$} & \multirow{2}{*}{$\begin{array}{l}\text { Total } \\
\text { No. }^{+}\end{array}$} & \multicolumn{2}{|c|}{ HPV (-) } & \multicolumn{2}{|c|}{ HPV (+) } \\
\hline & & & & & & & $\begin{array}{l}\text { Recur- } \\
\text { rence (-) }\end{array}$ & $\begin{array}{l}\text { Recur- } \\
\text { rence (+) }\end{array}$ & $\begin{array}{l}\text { Recur- } \\
\text { rence (-) }\end{array}$ & $\begin{array}{l}\text { Recur- } \\
\text { rence (+) }\end{array}$ \\
\hline Weber et al. & 1988 & USA & 6 & DNA/ISH & $46.2^{\mathrm{a}}$ & 21 & 5 & 0 & 9 & 7 \\
\hline Beck et al. & 1995 & USA & 6 & DNA/PCR & 32.2 & 22 & 10 & 0 & 2 & 10 \\
\hline Ogura et al. & 1996 & Japan & 5 & DNA/PCR & $\mathrm{N} / \mathrm{A}$ & 9 & 5 & 1 & 1 & 2 \\
\hline Bernauer et al. & 1997 & Germany & 6 & DNA/PCR & 37.6 & 21 & 13 & 1 & 5 & 2 \\
\hline Hwang et al. & 1998 & South Korea & 6 & DNA/PCR & 24.0 & 36 & 31 & 2 & 1 & 2 \\
\hline Kraft et al. & 2001 & Switzerland & 5 & DNA/PCR\&ISH & $\mathrm{N} / \mathrm{A}$ & 25 & 19 & 5 & 1 & 0 \\
\hline Jenko et al. & 2011 & Slovenia & 5 & DNA/PCR & $\mathrm{N} / \mathrm{A}$ & 66 & 37 & 9 & 15 & 5 \\
\hline Giotakis et al. & 2012 & Greece & 6 & DNA/PCR & $\geq 48.0^{\mathrm{b}}$ & 32 & 17 & 5 & 6 & 4 \\
\hline Roh et al. & 2016 & South Korea & 6 & DNA/PCR & 40.6 & 54 & 39 & 7 & 8 & 0 \\
\hline Lin et al. & 2016 & China & 5 & DNA/PCR & $\mathrm{N} / \mathrm{A}$ & 28 & 9 & 4 & 3 & 12 \\
\hline Husain et al. & 2020 & Malaysia & 5 & DNA/PCR & $\mathrm{N} / \mathrm{A}$ & 41 & 23 & 5 & 11 & 2 \\
\hline Fulla et al. & 2020 & Spain \& Poland & 6 & DNA/PCR & 70.1 & 76 & 56 & 16 & 3 & 1 \\
\hline Frasson et al. & 2020 & Italy & 6 & DNA/PCR & 35.7 & 55 & 14 & 7 & 25 & 9 \\
\hline $\begin{array}{l}\text { Pahler vor der } \\
\text { Holte et al. }\end{array}$ & 2021 & Germany & 6 & DNA/PCR & 37.2 & 106 & 49 & 10 & 31 & 16 \\
\hline
\end{tabular}

Abbreviations: NOS, Newcastle-Ottawa scale; HPV, human papillomavirus; F/U, follow-up; PCR, polymerase chain reaction; ISH, in situ hybridisation; $\mathrm{N} / \mathrm{A}$, not available. ${ }^{\dagger}$ Total number of sinonasal inverted papilloma cases with adequate information. ${ }^{\mathrm{a}}$ Data were extracted from selective cases for which detailed information was available. ${ }^{\mathrm{b}}$ This study reported that all patients had a minimum follow-up of 4 years.

\section{Results}

Literature search and study characteristics

The database search using the keywords and search strategies described above revealed 1,912 articles, 283 of which were excluded owing to duplication. After title and abstract screening, we excluded 1,584 articles and read the full text of 45 articles. Finally, 14 articles involving 592 patients with SNIP were included in our meta-analysis ${ }^{(10-23)}$. The process of study selection is presented in Figure 1. Among the 14 studies, nine were conducted in Western countries (USA, Germany, Switzerland, Slovenia, Greece, Spain, Poland, and Italy) and five in Eastern countries (South Korea, Japan, China, and Malaysia). The NOS scores of the included studies ranged from 5 to 6 , and the publication year ranged from 1988 to 2021 . All the enrolled studies were hospital-based. Twelve studies detected HPV DNA using polymerase chain reaction (PCR), one provided data based on in situ hybridisation (ISH) results, and one used both detection methods. The characteristics of the included studies are summarised in Table 1. Among a total of 592 patients with SNIP, 193 (32.6\%) were positive for HPV DNA. Tumour recurrence was observed in 144 patients (24.3\%).

\section{Association between the HPV positivity and recurrence of SNIP}

Since all included studies provided information on both the de- tection rate of HPV DNA and tumour recurrence in patients with SNIP, we included those for the meta-analysis to investigate the association between HPV positivity and recurrence. As there was no significant level of heterogeneity between the included studies $\left(I^{2}=37 \%\right)$, the fixed effects model was used. We found that HPV positivity was significantly associated with an increased risk of SNIP recurrence $(\mathrm{OR}=2.19,95 \% \mathrm{Cl}=1.37-3.50$; Figure 2$)$.

\section{Subgroup analysis}

We additionally performed a subgroup analysis of the association between HPV positivity and SNIP recurrence according to the HPV detection method, geographic region of studies, and publication year of studies. We divided the studies into two groups, the PCR and ISH, based on the HPV detection method. PCR group $(\mathrm{OR}=2.12,95 \% \mathrm{Cl}=1.32-3.40)$, but not ISH group $(\mathrm{OR}=$ $3.50,95 \% \mathrm{Cl}=0.37-33.29$ ), showed significantly higher odds in HPV-positive cases than in HPV-negative cases (Figure 3). One study using both PCR and ISH was included in both PCR and ISH groups. In the subgroup analysis according to the geographic region of the studies, the presence of HPV DNA was significantly associated with a high prevalence of in studies from Western countries $(\mathrm{OR}=1.94,95 \% \mathrm{Cl}=1.14-3.29)$, but not in those from Eastern countries $(\mathrm{OR}=3.57,95 \% \mathrm{Cl}=0.73-17.50$; Figure 4). Notably, studies conducted in Eastern countries $\left(I^{2}=55 \%\right)$ exhibited a higher pooled OR and heterogeneity than studies from 


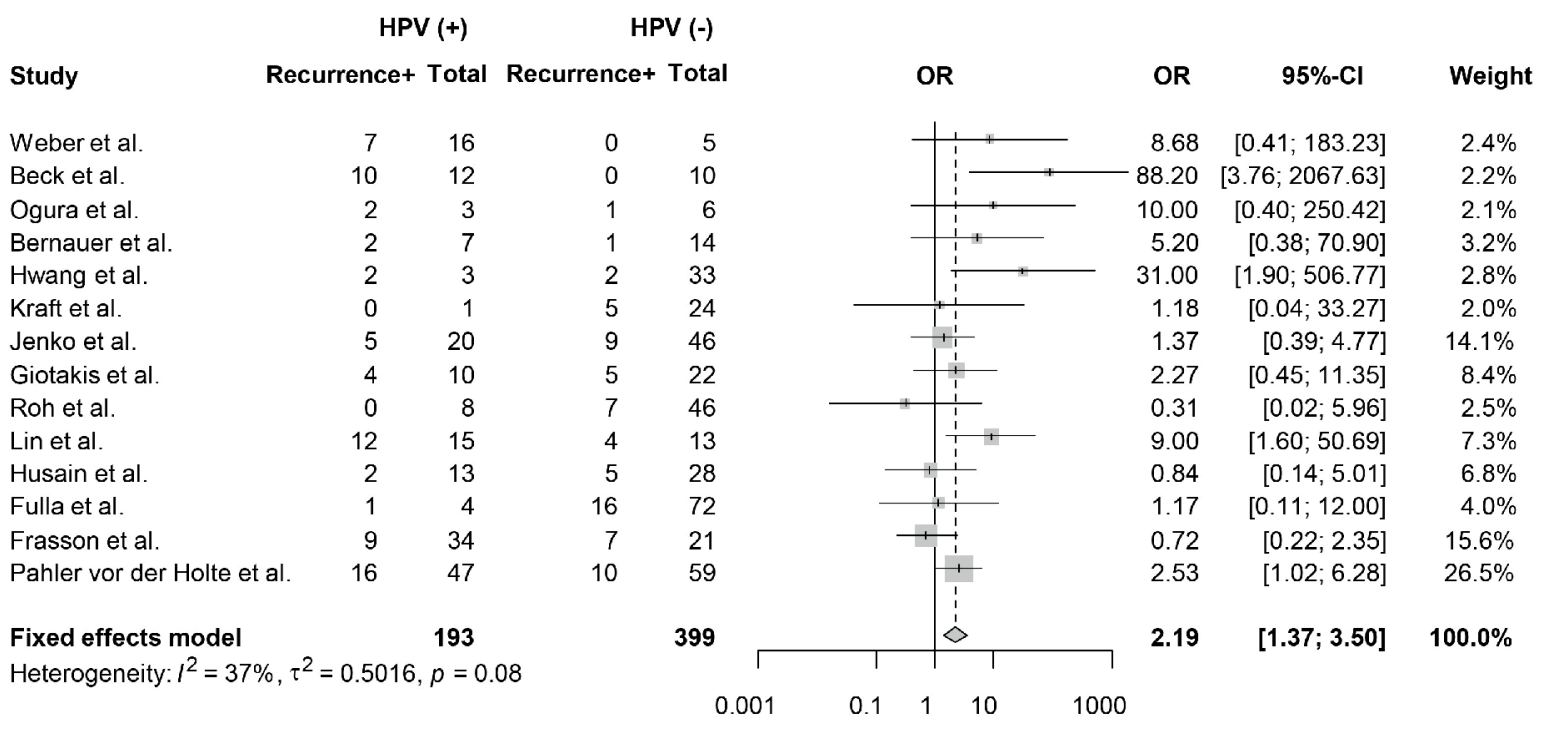

Figure 2. The pooled OR of the recurrence of sinonasal inverted papilloma associated with human papillomavirus infection. The calculation was based on the fixed effects model. The results are expressed as pooled ORs and 95\% Cls. HPV, human papillomavirus; OR, odds ratio; $\mathrm{Cl}$, confidence interval.

\section{Studies using PCR}

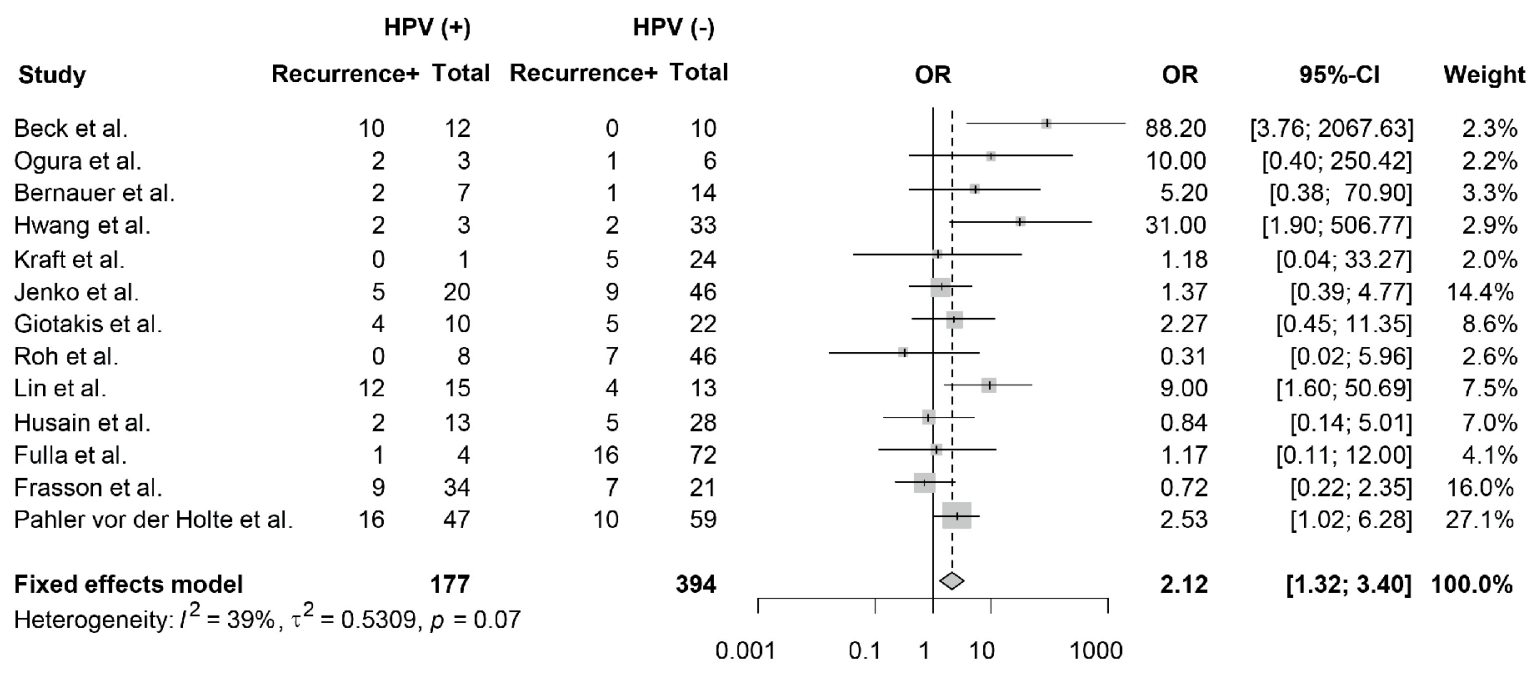

\section{Studies using ISH}

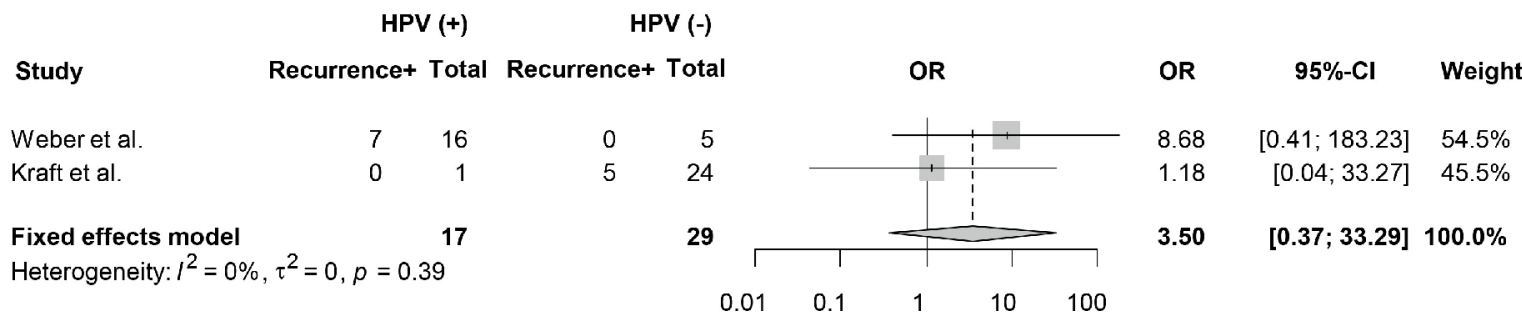

Figure 3. Subgroup analysis based on detection method of human papillomavirus DNA. Studies were divided into two subgroups ('PCR' and 'ISH') according to the HPV detection method. The calculation for each subgroup was based on the fixed effects model. The results are expressed as pooled ORs and 95\% Cls. HPV, human papillomavirus; OR, odds ratio; $\mathrm{Cl}$, confidence interval; PCR, polymerase chain reaction; ISH, in situ hybridisation. 


\section{Western countries}

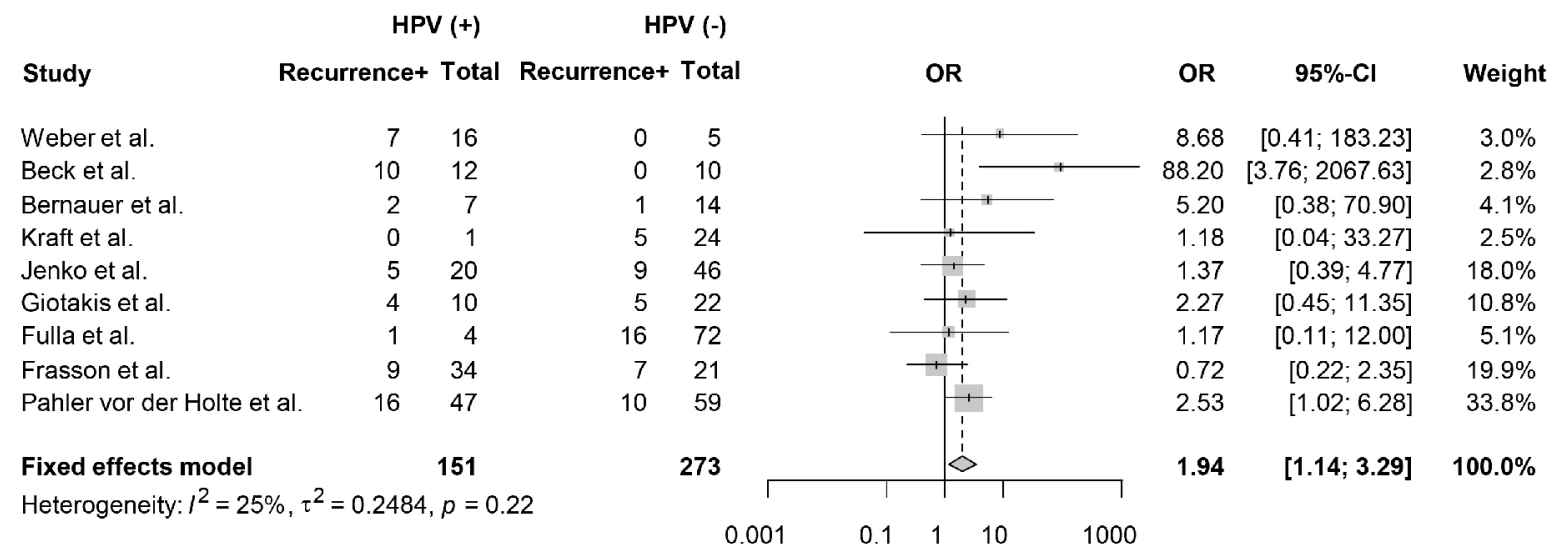

\section{Eastern countries}

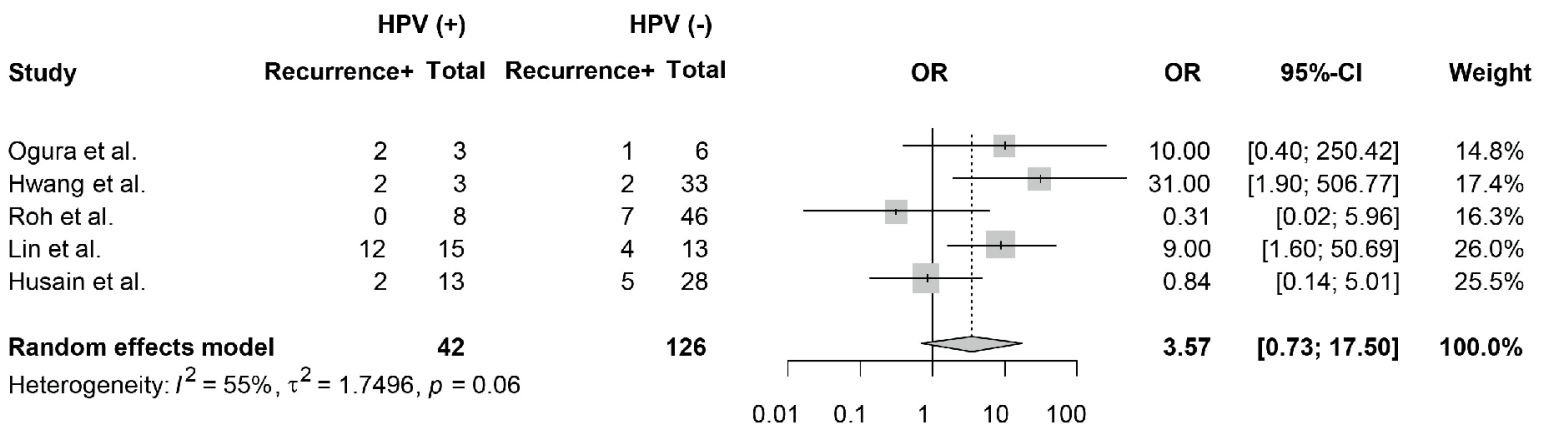

Figure 4. Subgroup analysis based on the geographic region of studies. Studies are divided into two subgroups ('Western countries' and 'Eastern countries') according to the geographic region of studies. The calculation for the 'Western countries' group and 'Eastern countries' group was based on the fixed effects model and random effects model, respectively. The results are expressed as pooled ORs and 95\% Cls. HPV, human papillomavirus; OR, odds ratio; $\mathrm{Cl}$, confidence interval.

Western countries $\left(I^{2}=25 \%\right)$. When we divided the total studies into pre-2000 and post-2000 groups according to the publication date, a significant association between HPV infection and risk of recurrence was observed in both the pre-2000 group (OR $=15.47,95 \% \mathrm{Cl}=4.16-57.58)$ and post -2000 groups $(\mathrm{OR}=1.65$ $95 \% \mathrm{Cl}=1.00-2.72$; Figure 5). Since information on the followup period for all study subjects was available in nine studies, we divided these studies into two subgroups based on the mean follow-up period: short-term ( $<3$ years) and long-term ( $\geq 3$ years) follow-up groups. We found that significantly higher ORs of tumour recurrence remained stable in long-term follow-up group $(\mathrm{OR}=2.32,95 \% \mathrm{Cl}=1.17-4.59)$, but not in short-term follow up group $(\mathrm{OR}=2.01,95 \% \mathrm{Cl}=0.72-5.63$; Figure 6$)$.

\section{Assessment of publication bias and sensitivity analysis} Visual inspection of funnel plots revealed symmetry of the included studies (Sup-Figure 1). Egger's regression test also suggested no significant evidence of publication bias $(t=0.76, P=$ 0.4611).
Next, we evaluated the stability of the results through sensitivity analysis. The corresponding pooled ORs were not substantially altered when single studies were sequentially removed, with pooled ORs ranging between 1.96 and 2.69 , implying that the results of the meta-analysis were stable (Sup-Figure 2 ).

\section{Discussion}

Although the contribution of HPV infection to the pathogenesis of SNIP has drawn considerable attention and has been widely investigated, previous results regarding the relationship between HPV infection and SNIP recurrence have been controversial. In the present meta-analysis, we found that HPV infection was significantly associated with a high risk of SNIP recurrence. We also found that this significant association remained stable in subgroup analysis according to publication year of studies. These results suggest that HPV testing needs to be considered in the management of SNIP.

The pathological role of HPV has been primarily identified in cervical cancer ${ }^{(7)}$. Infection with high-risk HPV and subsequent 


\section{Pre-2000}

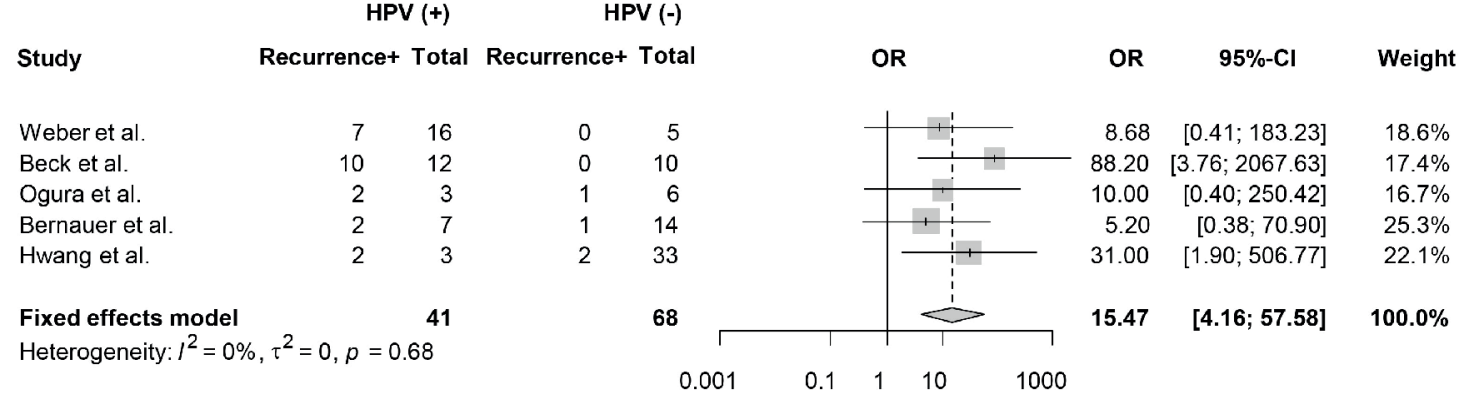

\section{Post-2000}

\begin{tabular}{|c|c|c|c|c|c|c|c|c|c|}
\hline Study & Recurrence+ & Total & \multicolumn{2}{|c|}{ Recurrence+ Total } & \multicolumn{2}{|c|}{ OR } & OR & $95 \%=\mathrm{Cl}$ & Weight \\
\hline Kraft et al. & 0 & 1 & 5 & 24 & & & 1.18 & {$[0.04 ; 33.27]$} & $2.3 \%$ \\
\hline Jenko et al. & 5 & 20 & 9 & 46 & & 4 & 1.37 & {$[0.39 ; 4.77]$} & $16.1 \%$ \\
\hline Giotakis et al. & 4 & 10 & 5 & 22 & & & 2.27 & {$[0.45 ; 11.35]$} & $9.7 \%$ \\
\hline Roh et al. & 0 & 8 & 7 & 46 & & & 0.31 & {$[0.02 ; 5.96]$} & $2.9 \%$ \\
\hline Lin et al. & 12 & 15 & 4 & 13 & & +1 & 9.00 & {$[1.60 ; 50.69]$} & $8.4 \%$ \\
\hline Husain et al. & 2 & 13 & 5 & 28 & & 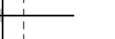 & 0.84 & {$[0.14 ; 5.01]$} & $7.8 \%$ \\
\hline Fulla et al. & 1 & 4 & 16 & 72 & & +1 & 1.17 & {$[0.11 ; 12.00]$} & $4.6 \%$ \\
\hline Frasson et al. & 9 & 34 & 7 & 21 & $\longrightarrow$ & $\frac{1}{1}$ & 0.72 & {$[0.22 ; 2.35]$} & $17.9 \%$ \\
\hline Pahler vor der Holte et al. & 16 & 47 & 10 & 59 & & \begin{tabular}{|l}
1 \\
1
\end{tabular} & 2.53 & {$[1.02 ; 6.28]$} & $30.4 \%$ \\
\hline Fixed effects model & & 152 & & 331 & & & 1.65 & {$[1.00 ; 2.72]$} & $100.0 \%$ \\
\hline \multirow{2}{*}{\multicolumn{5}{|c|}{ Heterogeneity: $I^{2}=7 \%, \tau^{2}=0.0452, p=0.38$}} & $\Gamma$ & & & & \\
\hline & & & & & 0.51 & 2 & & & \\
\hline
\end{tabular}

Figure 5. Subgroup analysis based on the publication date of studies. Studies were divided into two subgroups ('Pre-2000' and 'Post-2000') according to the publication year. The calculation for each subgroup was based on the fixed effects model. The results are expressed as pooled ORs and 95\% Cls. HPV, human papillomavirus; OR, odds ratio; $\mathrm{Cl}$, confidence interval.

viral DNA integration are known to drive the activation of oncogenes and carcinogenesis in the cervix ${ }^{(5)}$. Currently, testing for high-risk HPV DNA in cervical cytologic samples is routinely performed in women. In addition, prophylactic HPV vaccines have shown high efficacy in protection against cervical cancer (7). The impact of HPV infection on human health has also been extensively studied in oropharyngeal cancer. Virus-driven oropharyngeal SCCa shows distinct epidemiology and molecular mechanisms for cancer development compared to oropharyngeal SCCa driven by other carcinogens, such as smoking and alcohol ${ }^{(9)}$. HPV-positive oropharyngeal SCCa is characterised by p16 overexpression, whereas HPV-negative oropharyngeal SCCa has frequent $\mathrm{p} 53$ mutation ${ }^{(9,29)}$. Although the exact mechanism is unclear, patients with HPV-positive oropharyngeal SCCa show a better prognosis compared to those with HPV-negative cancer ${ }^{(30)}$. Similarly, it appears plausible that HPV-positive SNIP may have distinct clinical and molecular features compared to HPVnegative counterparts.

The high risk of tumour recurrence is a major concern in the treatment of SNIP. Given that the current treatment of choice for SNIP is radical surgery, incomplete resection of the tumour and predisposed mucosa may underlie tumour recurrence. In the present study, we revealed that HPV infection may be a risk factor for the recurrence of SNIP. Given that integration of HPV viral sequences into the host cellular DNA promotes tumour growth ${ }^{(5)}$, the remaining sinonasal mucosa infected by HPV may predispose to tumour recurrence. A significant association between HPV infection and malignant transformation of SNIP was similarly observed in a recent meta-analysis ${ }^{(31)}$, suggesting that HPV infection is also involved in the progression from SNIP to SCCa. Further prospective studies with large cohorts are required to clarify the causative role of HPV infection in the recurrence of SNIP. In addition, the molecular mechanism underlying the pathological role of HPV in SNIP should be addressed in future studies.

The HPV detection rate in SNIPs was highly variable, ranging from $0 \%$ to $100 \%$ in previous studies ${ }^{(32)}$. This discrepancy in HPV prevalence between studies may be derived from the different sensitivities of HPV detection methods ${ }^{(33,34)}$. Others also suggest that HPV prevalence may vary across geographic regions ${ }^{(35)}$. In addition, since the sensitivity of detection methods has improved gradually, the publication date of studies may influence the results. Thus, we performed subgroup analyses to examine whether the association of HPV infection with SNIP recurrence 
Short-term follow-up (<3 years)

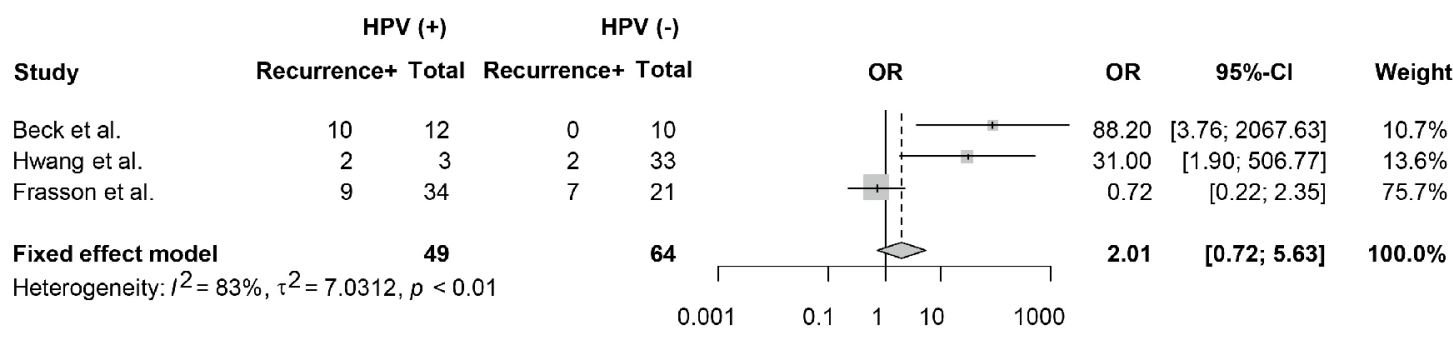

\section{Long-term follow-up ( $\geq 3$ years)}

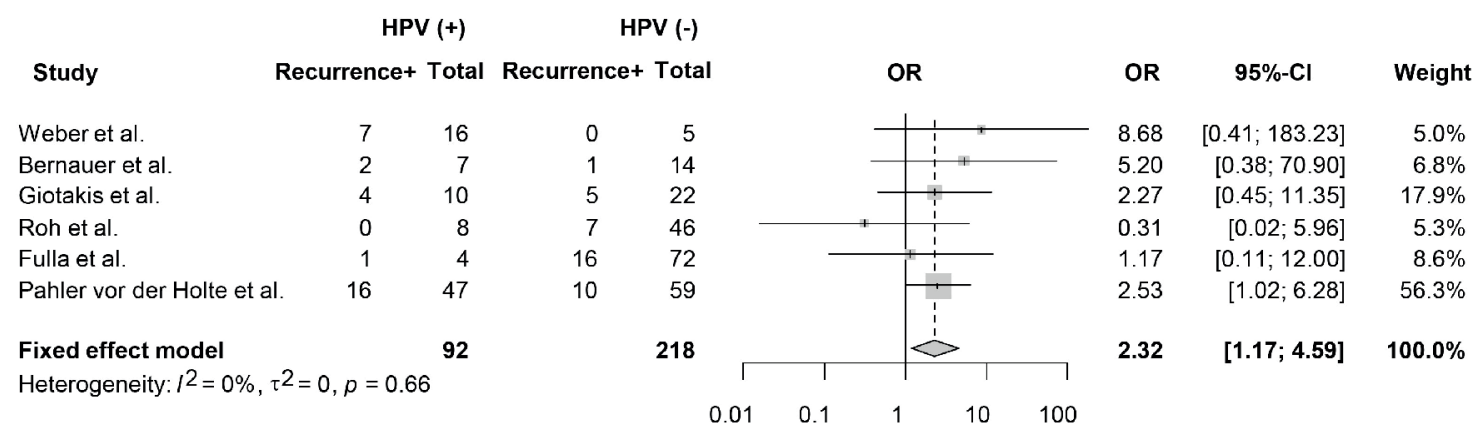

Figure 6. Subgroup analysis based on the mean follow-up period. Studies were divided into two subgroups ('short-term' and 'long-term') according to the mean follow-up period. The calculation for each subgroup was based on the fixed effects model. The results are expressed as pooled ORs and $95 \%$ Cls. HPV, human papillomavirus; OR, odds ratio; $\mathrm{Cl}$, confidence interval.

is affected by these possible confounding factors. We found that a significant association was observed in studies using PCR, but not in those using ISH. Considering that only two studies used ISH, more data are needed to clarify the results. We also observed that studies from Eastern countries showed no significant association between HPV infection and the recurrence of SNIP. These results may be due to several factors. First, the higher heterogeneity between studies may affect the results. Second, dominant HPV genotype may be different between geographic regions. Further studies would be required to investigate whether the association between HPV infection and the recurrence of SNIP relies on race or geographic factors.

It has been shown that the pathogenicity of HPV differs among genotypes. Infection by certain HPV genotypes may lead to malignant progression due to uncontrolled activation of viral oncogenes. Specifically, low-risk HPV genotypes (HPV 6 or HPV 11) cause papillomatous lesions but generally do not lead to malignancy, whereas the risk of malignant transformation is significantly increased in high-risk HPV genotypes (HPV 16 or HPV 18). A recent study reported that both low-risk and highrisk HPV genotypes were identified in SNIP ${ }^{(22)}$. However, little is known about HPV genotypes that are responsible for the recurrence of SNIP. Intriguingly, a previous study suggested that recurrent SNIP cases showed an increased rate of infection with low-risk HPV genotypes, particularly HPV $6^{(23)}$. To develop effective HPV vaccines or HPV screening methods for SNIP, the specific genotypes that cause the recurrence of SNIP need to be elucidated in future studies.

This study has several limitations. First, the effects of possible confounding factors, such as surgical approach and tumour location, were not adjusted in the analysis. Second, we excluded articles with incomplete information or those written in foreign languages, which may have biased the results. Third, although we performed subgroup analysis according to the mean followup period of all study subjects, detailed data regarding followup periods in HPV-positive and HPV-negative cases respectively were limited. Therefore, further prospective studies with a long-term follow-up of both HPV-positive and HPV-negative groups would be required to validate the current results. Fourth, the majority of included studies had a retrospective design, which generally has a lower level of evidence than prospective cohort studies. Despite these limitations, our study is the first meta-analysis to provide valuable information regarding the link between HPV infection and SNIP recurrence. 


\section{Conclusion}

The current meta-analysis demonstrated that HPV infection was significantly associated with the recurrence of SNIP. Furthermore, this significant association was consistently observed in subgroup analysis according to publication year of studies. These findings suggest the contribution of HPV infection to the pathogenesis of SNIP, thus supporting the need to consider HPV infection in the diagnosis and treatment of patients with SNIP.

\section{Authorship contribution}

M-SR, J-HY, and H-JC designed the study. M-SR and H-JC performed the literature search and extracted the data. M-SR and C-HK analysed the data. M-SR and $\mathrm{H}-\mathrm{JC}$ wrote the manuscript. All authors reviewed the manuscript.

\section{Conflict of interest}

The authors declare no conflicts of interests.

\section{Funding}

This research was supported by a grant of the Korea Health Technology R\&D Project through the Korea Health Industry Development Institute (KHIDI), funded by the Ministry of Health \& Welfare, Republic of Korea (grant number: HV20C0205), and Basic Science Research Program through the National Research Foundation of Korea (NRF) funded by the Ministry of Education (2021R1I1A1A01047571).

\section{References}

1. Govindaraj S, Wang HL. Does human papilloma virus play a role in sinonasal inverted papilloma? Curr Opin Otolaryngol Head Neck Surg. 2014;22(1):47-51

2. Mirza S, Bradley PJ, Acharya A, Stacey M, Jones NS. Sinonasal inverted papillomas: recurrence, and synchronous and metachronous malignancy. J Laryngol Otol. 2007;121(9):857-864.

3. Wang MJ, Noel JE. Etiology of sinonasal inverted papilloma: A narrative review. World J Otorhinolaryngol Head Neck Surg. 2017;3(1):54-58

4. Doorbar J. The papillomavirus life cycle. Clin Virol. 2005;32 Suppl 1:S7-15.

5. Moody CA, Laimins LA. Human papillomavirus oncoproteins: pathways to transformation. Nat Rev Cancer. 2010;10(8):550-560.

6. Munger $K$, Baldwin A, Edwards KM et al. Mechanisms of human papillomavirus-induced oncogenesis. J Virol. 2004;78(21):11451-11460

7. Crosbie EJ, Einstein MH, Franceschi S, Kitchener HC. Human papillomavirus and cervical cancer. Lancet. 2013;382(9895):889899.

8. Castellsague X, Alemany L, Quer M, et al. HPV Involvement in Head and Neck Cancers: Comprehensive Assessment of Biomarkers in 3680 Patients. J Natl Cancer Inst. 2016;108(6):djv403.

9. Pytynia KB, Dahlstrom KR, Sturgis EM. Epidemiology of HPV-associated oropharyngeal cancer. Oral Oncol. 2014:50(5):380-386.

10. Weber RS, Shillitoe EJ, Robbins KT, et al. Prevalence of human papillomavirus in inverted nasal papillomas. Arch Otolaryngol Head Neck Surg. 1988;114(1):23-26.

11. Beck JC, McClatchey KD, Lesperance MM, Esclamado RM, Carey TE, Bradford CR. Presence of human papillomavirus predicts recurrence of inverted papilloma. Otolaryngol Head Neck Surg. 1995:113(1):49-55.
12. Ogura H, Fukushima K, Watanabe S. A high prevalence of human papillomavirus DNA in recurrent nasal papillomas. J Med Microbiol. 1996;45(3):162-166

13. Bernauer HS, Welkoborsky HJ, Tilling A Amedee RG, Mann WJ. Inverted papillomas of the paranasal sinuses and the nasal cavity: DNA indices and HPV infection. Am J Rhinol. 1997:11(2):155-160.

14. Hwang CS, Yang HS, Hong MK. Detection of human papillomavirus (HPV) in sinonasal inverted papillomas using polymerase chain reaction (PCR). Am J Rhinol. 1998;12(5):363-366

15. Kraft $M$, Simmen D, Casas R, Pfaltz M Significance of human papillomavirus in sinonasal papillomas. J Laryngol Otol. 2001;115(9):709-714.

16. Jenko K, Kocjan B, Zidar N, et al. In inverted papillomas HPV more likely represents incidental colonization than an etiological factor. Virchows Arch. 2011;459(5):529-538.

17. Giotakis E, Gomatos IP, Alevizos L, et al. Apoptotic and proliferative status in HPV (+) and HPV (-) inverted papilloma patients. Correlation with local recurrence and clinicopathological variables. Pathol Res Pract. 2012;208(6):338-343.

18. Roh HJ, Mun SJ, Cho KS, Hong SL. Smoking, not human papilloma virus infection, is a risk factor for recurrence of sinonasal inverted papilloma. Am J Rhinol Allergy. 2016;30(2):79-82

19. Lin H, Lin D, Xiong XS. Roles of human papillomavirus infection and stathmin in the pathogenesis of sinonasal inverted papilloma. Head Neck. 2016;38(2):220-224.

20. Husain S, Ishak NL, Gendeh B, et al. A CrossSectional Study on Sinonasal Inverted Papilloma: Does Human Papilloma Virus Play a Role in Its Etiology?. Authorea. 2020 doi: 10.22541/au.158678550.00655404.

21. Fulla M, Szafarowski T, Frias-Gomez J, et al. Human Papillomavirus and Factors Associated with Recurrence in Sinonasa Inverted Papillomas from Poland and Spain.
Head Neck Pathol. 2020;14(3):758-767.

22. Frasson G, Cesaro S, Cazzador D, et al. High prevalence of human papillomavirus infection in sinonasal inverted papilloma: a single-institution cohort of patients. Int Forum Allergy Rhinol. 2020;10(5):629-635.

23. Paehler Vor der Holte A, Fangk I, Glombitza S, Wilkens L, Welkoborsky HJ. Impact of human papillomaviruses (HPV) on recurrence rate and malignant progression of sinonasal papillomas. Cancer Med. 2021;10(2):634-641.

24. Moher D, Liberati A, Tetzlaff J, Altman DG, Group P. Preferred reporting items for systematic reviews and meta-analyses: the PRISMA statement. PLoS Med. 2009;6(7):e1000097.

25. Stang A. Critical evaluation of the Newcastle-Ottawa scale for the assessment of the quality of nonrandomized studies in meta-analyses. Eur J Epidemiol. 2010;25(9):603-605.

26. Wan X, Wang W, Liu J, Tong T. Estimating the sample mean and standard deviation from the sample size, median, range and/or interquartile range. BMC Med Res Methodol. 2014;14:135.

27. Egger M, Davey Smith G, Schneider M, Minder C. Bias in meta-analysis detected by a simple, graphical test. BMJ. 1997;315(7109):629-634.

28. Lubin JH, Boice JD, Jr. Lung cancer risk from residential radon: meta-analysis of eight epidemiologic studies. J Natl Cancer Inst. 1997;89(1):49-57.

29. Taberna M, Mena M, Pavon MA, Alemany L, Gillison ML, Mesia R. Human papillomavirus-related oropharyngeal cancer. Ann Oncol. 2017;28(10):2386-2398.

30. Ang KK, Harris J, Wheeler R, et al. Human papillomavirus and survival of patients with oropharyngeal cancer. N Engl J Med. 2010;363(1):24-35

31. Stepp WH, Farzal Z, Kimple AJ, et al. HPV in the malignant transformation of sinonasal inverted papillomas: A meta-analysis. 
Int Forum Allergy Rhinol. 2021;11(10):1461 1471.

32. Syrjanen K, Syrjanen S. Detection of human papillomavirus in sinonasal papillomas: systematic review and meta-analysis Laryngoscope. 2013;123(1):181-192.

33. Buchwald C, Franzmann MB, Jacobsen GK, Lindeberg H. Human papillomavirus (HPV) in sinonasal papillomas: a study of 78 cases using in situ hybridization and polymerase chain reaction. Laryngoscope. 1995;105(1):66-71.

34. Furuta $Y$, Shinohara $T$, Sano $K$, et al. Molecular pathologic study of human papillomavirus infection in inverted pap- illoma and squamous cell carcinoma of the nasal cavities and paranasal sinuses. Laryngoscope. 1991;101(1 Pt 1):79-85.

35. Crow JM. HPV: The global burden. Nature. 2012;488(7413):S2-3.
Hyung-Ju Cho

Department of Otorhinolaryngology Yonsei University College of Medicine 50-1 Yonsei-ro

Seodaemun-gu

Seoul, 03722

Republic of Korea

Tel: +82-2-228-3609

E-mail: hyungjucho@yuhs.ac 


\section{SUPPLEMENTARY MATERIAL}

Supplementary Table 1. Search strategy.

\begin{tabular}{|c|c|}
\hline Source & Search Terms \\
\hline Pubmed & $\begin{array}{l}\text { ("Papilloma, Inverted"[MeSH] OR "inverted papilloma"[All Fields]) AND (nasal[All Fields]) OR sinonasal[All Fields]) AND } \\
\text { ("Papillomaviridae"[MeSH] OR "human papillomavirus"[All Fields] OR HPV[All Fields]) }\end{array}$ \\
\hline Web of Science & ALL=("inverted papilloma" AND (nasal or sinonasal) AND ("human papillomavirus" OR "HPV")) \\
\hline Google Scholar & "inverted papilloma” AND (nasal or sinonasal) AND ("human papillomavirus" OR "HPV") \\
\hline The Cochrane Library & $\begin{array}{l}\text { \#1: "inverted papilloma” } \\
\text { \#2: MeSH descriptor: [Papilloma, Inverted] explode all trees } \\
\text { \#3: nasal OR sinonasal } \\
\text { \#4: MeSH descriptor: [Papillomaviridae] explode all trees } \\
\text { \#5: "human papillomavirus" OR HPV } \\
\text { \#6: \#1 OR \#2 } \\
\text { \#7: \#4 OR \#5 } \\
\text { \#5: \#3 AND \#6 AND \#7 }\end{array}$ \\
\hline
\end{tabular}

Supplementary Table 2. Quality assessment of included studies.

\begin{tabular}{|c|c|c|c|c|c|c|c|}
\hline \multirow[t]{2}{*}{ Study } & \multicolumn{3}{|c|}{ Selection } & \multirow{2}{*}{$\begin{array}{l}\text { Comparability } \\
\text { Comparability } \\
\text { of cohorts on } \\
\text { the basis of } \\
\text { the design or } \\
\text { analysis } \\
\text { (maximum **) }\end{array}$} & \multicolumn{2}{|c|}{ Outcome } & \multirow[t]{2}{*}{ Total score } \\
\hline & $\begin{array}{c}\text { Representative- } \\
\text { ness }\end{array}$ & $\begin{array}{l}\text { Selection of the } \\
\text { non-exposed } \\
\text { cohort }\end{array}$ & $\begin{array}{c}\text { Ascertainment } \\
\text { of exposure }\end{array}$ & & $\begin{array}{l}\text { Assessment of } \\
\text { outcome }\end{array}$ & $\begin{array}{l}\text { Adequacy of } \\
\text { follow-up }\end{array}$ & \\
\hline Weber et al. & * & * & * & * & * & * & 6 \\
\hline Beck et al. & $*$ & * & * & * & * & * & 6 \\
\hline Ogura et al. & $*$ & * & * & * & * & - & 5 \\
\hline Bernauer et al. & * & * & * & * & * & * & 6 \\
\hline Hwang et al. & * & * & * & * & * & * & 6 \\
\hline Kraft et al. & * & * & * & * & * & - & 5 \\
\hline Jenko et al. & * & * & * & * & * & - & 5 \\
\hline Giotakis et al. & $*$ & $*$ & * & * & * & * & 6 \\
\hline Roh et al. & * & * & * & $*$ & * & $*$ & 6 \\
\hline Lin et al. & * & * & * & * & * & - & 5 \\
\hline Husain et al. & * & * & * & * & * & - & 5 \\
\hline Fulla et al. & * & * & * & * & * & * & 6 \\
\hline Frasson et al. & * & * & * & * & * & * & 6 \\
\hline $\begin{array}{l}\text { Pahler vor der } \\
\text { Holte et al. }\end{array}$ & * & $*$ & * & * & * & * & 6 \\
\hline
\end{tabular}




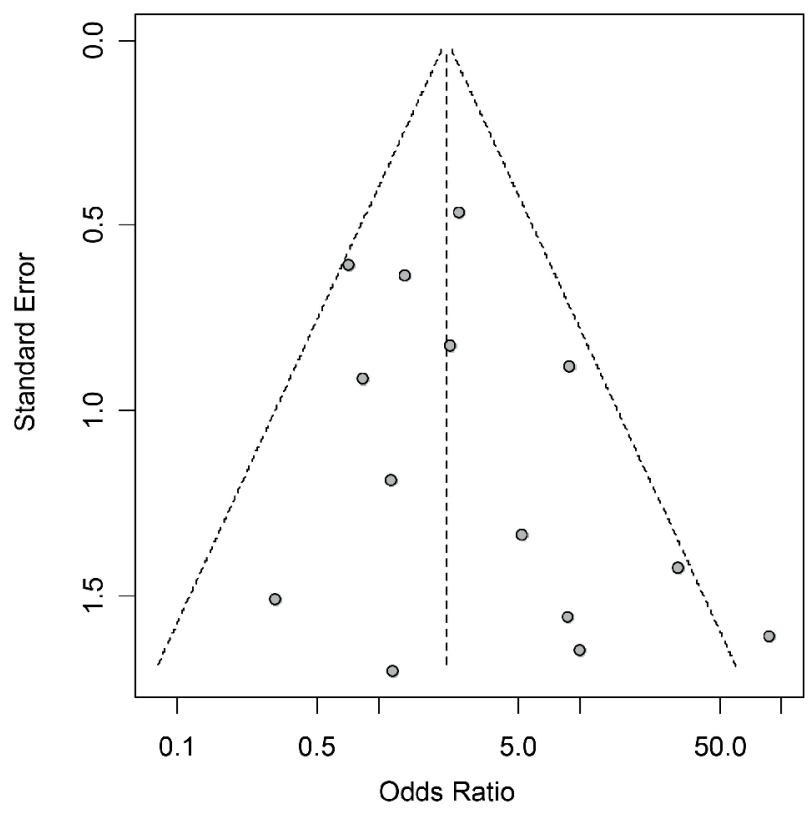

Supplementary Figure 1. The funnel plot for assessing the publication

bias.

\section{Study}

Omitting Weber et al.

Omitting Beck et al.

Omitting Ogura et al.

Omitting Bernauer et al.

Omitting Hwang et al.

Omitting Kraft et al.

Omitting Jenko et al.

Omitting Giotakis et al.

Omitting Roh et al.

Omitting Lin et al.

Omitting Husain et al.

Omitting Fulla et al.

Omitting Frasson et al.

Omitting Pahler vor der Holte et al.

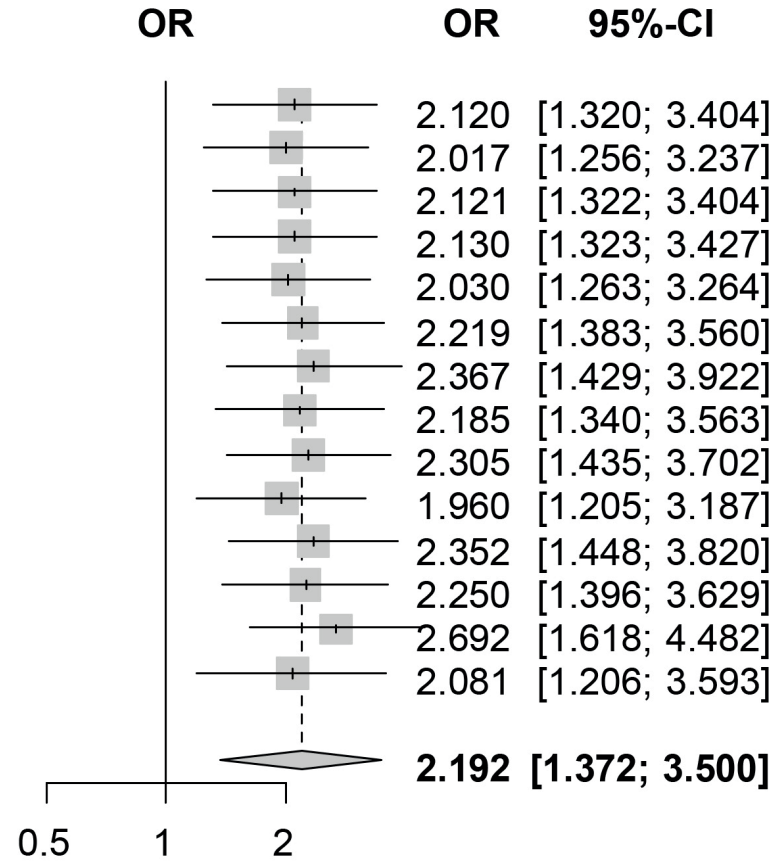

Supplementary Figure 2. Sensitivity analysis of the meta-analysis. A forest plot for the sensitivity analysis is presented. The calculation was based on the fixed effects model. The results are expressed as odds ratios and $95 \%$ confidence intervals. OR, odds ratio; $\mathrm{Cl}$, confidence interval. 